\title{
Forecasting Interregional Migration. Three Methods Used in Swedish Planning
}

\author{
EINAR HOLM \\ OLA NYGREN
}

\section{Backround}

This paper deals with the modeling of regional migration in three different Swedish forecasting systems which are more or less official in character:

- county planning (hereafter CP)

- regional breakdown of the 1984 medium-term survey (hereafter MS), and

- forecasts forming the basis of housing and building planning (hereafter $\mathrm{HO}$ ).

First, the aims and objectives of the three different sets of models are outlined as well as their principal features. Next, the modeling of regional migration is described in more detail. Finally, some major results of the various alternatives are presented.

\section{Aims and objectives of the forecasts}

The objectives of county planning (CP) are basically two: to provide a foundation, first, for regional policy at central level and, secondly, for resource allocation in the public sector at regional level. County planning has an indicative emphasis centring on the description and analysis of the present situation and expected future trends as regards regional population and labor distribution. Links with instruments of regional policy are indirect only. CP-forecasts have inreasingly provided a basis for dimensioning decisions in the health and medical care sector, which are taken by the local county councils.

The purpose of medium-term surveys (MS) are, starting with certain economic and political objectives, to describe the economic policy measures needed to achieve the desired effects. The 1984 MS may be characterized as an analysis of requirements rather than a forecast. The MS is compiled by staff of the Ministry of Finance and is in the form of an expert group's report. The objectives surrounding its formulation are full employment, a stable value of money, equilibrium in payments to and from abroad, a »reasonably» balanced national budget and a greater yield for real investments than for financial ones. The Medium-Term Survey posits two further aims which are harder to interpret quantitatively, namely a more equitable distribution of living standards and balanced regional development.

The purpose of the breakdown by regions of the MS is, above all, to shed light on whether its regional implications are feasible, i.e. do not require unrealistic resource transfers between regions. Regional analysis was carried out by the Expert Group for Research on Regional Development (ERU). In presenting the regional breakdown (SOU 1984: 6), they laid emphasis on a comparison with CP, which follows the "bottom-up principle»: forecasts are prepared at local and regional levels and summarized at regional and national levels. Large deviations for individual regions may indicate that general economic development is creating imbalances in regional development. 
Estimates of housing demand (HO) are compiled regularly in connection with and as a basis for the MS. The estimates are primarily designed to provide the foundation for assessing investments and employment in the building and construction sector. These estimates were formerly based on a national econometric model in which trends in real income and relative housing costs were the principal explanatory factors.

In connection with the extensive study of housing policy at present being carried out by a Government Committee (the Housing Committee), it has been considered essential to undertake a more ambitious survey of housing demand. The fundamental idea in these studies is that housing units sited in different regions are not interchangeable. The present econometric model cannot take into account factors such as regional migration.

The results of the regionally-based assessments (Hårsman, Holmberg and Schéele, 1984) will be used, among other things, in shaping housing provision plans. Unlike CP and the MS, which are recurrent assessments, the HO report is an isolated occurrence.

\section{Description of three-model systems}

The county planning forecasting system was designed at the end of the $1960 \mathrm{~s}$. The core of the system - which also functions as a data base for regional statistics - is a model of the labor market. Forecasts of employment and population trends have been made annually since 1975 , and they normally cover a period of five to eight years. The regional classification is (with the exception of the Stockholm region) by municipality, i.e. forecasts are compiled for 260 areas. The county administrations are responsible for the forecasts, and the data collected arise to some extent from knowledge of local conditions, particularly of industry and trade. This also means that the summarized forecasts are not consistent with the national forecast carried out by the Central Bureau of Statistics (SCB).

The first stage in forecasting consists of a demographic projection. Initially, the population in the base year is divided by sex and one-year age classes. Factors effecting changes in the projection are out-migration and mortality. One fundamental assumption in the forecasting model is that as a rule, regardless of the situation in the labor market, people move in and out of a municipality to some extent. Statistics regarding emigration and mortality for the municipality in question during some historical period are used as input.

The supply of labor in the municipality is calculated by applying exogenously assessed employment participation rates in different age classes to the projected population and estimated out-migration rate. These calculations thus yield a projection of the number of persons gainfully employed in the municipality, and of the number of gainfully employed persons migrating out of it. The difference between the two, i.e., the number of gainfully employed persons remaining after outmigration, constitutes the supply of labor in the municipality.

Estimates of demand for labor are compiled for 35 different branches of the economy. The documentation used here includes the MS and planning information from companies, municipalities and government authorities. The estimates of labor demand are also based on analyses of changes that have taken place to date in employment and production in the branch concerned. They are drawn up with the assumption that present efforts of regional policy will continue unchanged.

Demand for labor in the various branches is summarized as an overall demand for labor, which is thereafter set against the overall supply of labor. For the calculation of actual employment potential for the population of a municipality, labor demand is therefore adjusted according to assumptions regarding persons commuting into and out of the municipality during the forecasting period. 
In the next stage of the forecasting work, the supply of and demand for labor are balances and, usually, a labor differential is obtained. Gross in-migration is estimated so as to achieve a balance between labor supply and demand.

In-migration is calculated by means of the average employment participation rate of the municipality's resident population. The numbers of persons moving into the municipality are added to the population remaining there, i.e., the projected population is reduced by a number corresponding to gross out-migration. After that the number of births is calculated and added to the estimated population.

Summing up, the following variables may be said to be exogenous in relation to the model, i.e., they are assumptions inserted into, or adjusted in, the model:

- mortarily rates

- fertility rates

- labor force participation rates

- demand for labor

- commuting in and out

- out-migration rates

- age structure of the in-migrating population.

The Medium-Term Survey starts with an econometric macromodel of the Swedish economy, partly broken down by region and partly determined by the estimated conditions of production for each region. The regional consequences of $\mathrm{CP}$ are obtained at the county level, mainly as a breakdown of results from the national model regarding the economy, employment and population. In the breakdown model, however, economic variables (production, productivity, investment, etc.) are used only for industry. A special breakdown technique is used here, mainly based on information on the productivity and profitability structure of the industrial work places in each region (SIND 1982: 3). For other branches of the economy and the public sector, the inter-regional distribution of employment is grounded entirely on CP-assessments.

The methods used mean that the regional distribution of labor demand diverges from $\mathrm{CP}$ only in industry, i.e., 30 percent of employment, whereas the national forecast for employment participation rates has been broken down to the county level. This means that 100 percent of the regional supply of labor diverges from the county planning assessment.

Another important difference from CP is that the sum of the regional demographic forecasts is consistent with the national demographic forecast produced by SCB, as regards both total population and age structure.

Finally, the regional MS estimates also contain an assessment of the directions of migration flows. This cannot directly be inferred from the county planning estimates.

Otherwise, MS uses the same model as CP for describing the links between the demand for labor and population trends: given unchanged local labor force participation, an increase in demand generates an increase in population. The model does not include any interdependence between demand and labor force participation (or commuting).

The breakdown refers to counties ( 24 regions). It is true that the estimates have been made at municipal level ( 260 areas), but distribution within the regions is entirely based on that of CP. The municipal level has been used primarily in order that the results of classifications other than the purely administrative - e.g. urban-rural, regional aid areas and so on - may be presented.

The housing demand estimates ( $\mathrm{HO}$ ) are essentially different from the two other models, in that they do not take into account the distribution of demand for labor in the population projection which constitutes the first stage of the model system. In addition, the classification of regions is somewhat less refined (11 


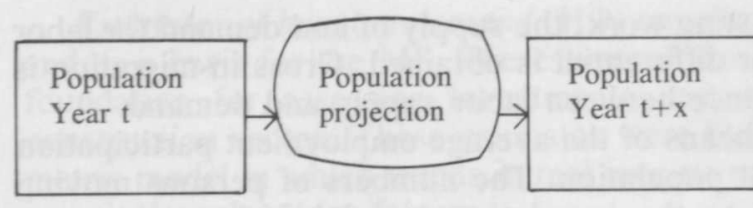

Figure 1 .

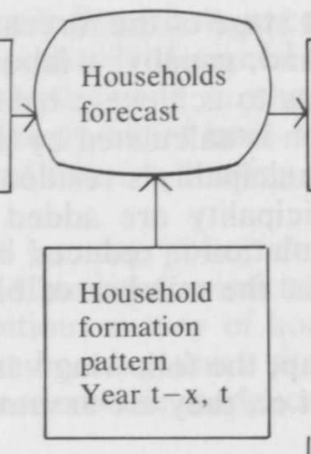

No. of households Year $\mathrm{t}+\mathrm{x}$

Historical patterns of decline

Housing construction needs



regions, largely aggregates of countries). The basic structure of the model is shown in Figure 1.

The population projection thus constitutes one of three calculation models in the system. The development of theory for the two other models has not gone nearly as far as they have for demographic forecasts.

The households forecast is based on the observed flows between various categories of household in the immediately preceding five-year period. These flows are subjected to restrictions based on the age structure of the population, migration and the numbers of births and deaths calculated in the population estimates (Hårsman and Scheele, 1982). In calculations of the decline in the housing stock ordinary life table techniques, dividing the housing units into age categories, are used. This calculation is a very unreliable component of the model system, since models of factors governing changes in housing stock are still very rudimentary in Sweden. This is partly because a great deal of "genuine» uncertainty is concealed in the demolition and re-building frequencies, which may alter substantially in the short term, e.g., as a result of government subsidies.

\section{Migration - theory and methods of estimation}

None of the models starts from an explicit theory of the causes of migration. The basic choice of theory, as well as the principal causal connections, must be inferred from the model structures and, to some extent, from the use made of the results.

The choice of model, methods and variables must of course be considered in the context of the relevant purpose. One of the purposes of CP and MS is to shed 
light on regional imbalances - a concept which is not explicitly defined in terms of one or more of the variables included.

Since the instruments of regional policy are principally directed towards industry, changes in the distribution of demand for labor are thought to have crucial significance as »indicators of imbalance». Another common indicator of this kind in the Swedish labor market is female labor force participation in different regions. A third is net migration, whereas the level of gross migrations is used more as an indicator of total mobility in the labor market (if a relevant regional classification and calibration period are chosen). Last but not least, the final result of the calculations - an age-distributed demographic forecast - is obviously highly relevant to the extent that the forecasts are the foundation for dimensioning decisions.

In the $\mathrm{HO}$ model, the latter is in practice the only variable which has any relevance at all, whereas migration is included only as an "auxiliary variable».

The partially divergent emphases of CP and MS are also reflected to some extent in methodology. Thus the means of calculating labor force participation in the MS are not particularly sophisticated, whereas migration estimates are more complex and accurate than in CP. As regards the $\mathrm{HO}$ model, it may be stated that its method of treating migration is in some respects overambitious: in the "specification of requirements", neither gross nor net migration are included, but the model goes as far as to estimate directed gross migration flows.

The county planning and Medium-Term Survey methods, as so far described, involve the following implicit assumptions, among others:

1. Out-migration is independent of the labor market within and outside the municipality.

2. Labor force participation is independent of the availability of jobs.

3. The level of in-migration is entirely dependent on the estimated number of employed persons in the municipality (corrected for commuting) and the exogenously assessed degree of gainful employment in the local population, but independent of employment and population in the country as a whole.

4. Every employed in-migrant brings with him a certain number of persons without jobs, i.e., all these are co-migrants of the employed individuals.

5. The whole labor market - i.e., all jobs and individuals - can in principle be reallocated every year to attain equilibrium, i.e., no distinction is made between residual employment, separation and acceptancy.

6. Every individual is assumed to be capable of taking and getting every job, i.e., labor sub-markets do not exist.

7. The migratory behavior of individuals is not influenced by any family connections.

This is not necessarily as far-fetched as it appears. A forecasting model does not improve with the incorporation of well-supported explanatory factors unless these in turn are easier to forecast. On the other hand, it is harder to justify the omission of apparent interdependences between factors already in the model. Moreover, there is an obvious difference between the model specifications generated by the desire for good (short-term) forecasts and the wish to obtain a relevant explanation. A blunt aggregated projection of trends often hits the mark better than a result from a more complex, disaggregated causal model. In a comparative survey of the results of a number of regional forecasting models, Ledent (1981) shows, among other things, that endogenous residual calculation of variables which, by definition, depend on other forecasted variables having similar values often leads to grossly incorrect results.

Some of the implications of those assumptions for the interpretation of the results reported later are as follows. 
- Omitting the feedback on labor force participation and the direct link between the employment level and in-migration probably results in an overestimate of the effects of changes in employment on migration. On the other hand, the fact that neither labor sub-markets nor separation and acceptancy are taken into account - even when the total demand level is unchanged - means that a great deal of migration resulting from labor market conditions does not emerge at all from the model. Such migration varies possibly less than, and probably differently in time and space from, migration caused by changes in demand. The net effects might then be that in-migration is overestimated at a time of expansion and underestimated in a recession. Moreover, the model will probably often show the "wrong» people to be migrating.

- The model ascribes the total effect of changes in the labor market to (net) in-migration, instead of treating effects on in-migration and out-migration respectively. This may to some extent be justified in that the connection between employment changes and out-migration are considerably weaker than the connection with in-migration. The net flows produced are thus, in a sense, more reliable than the gross flows.

- There is no explicit consideration whatsoever of the role of household formation as a cause of migration. Presumably, these vary less in time and space than those causes directly connected with the labor market. If so, one might state that migration prompted by household changes is gauged by out-migration rates and in-migrant proportions exogenous to the model. One problem, however, is that all demographic in-migration is regarded as co-migration. Owing to this, variations are exaggerated: in relation to employment, in-migration is excessive in expansive municipalities and insufficient in municipalities suffering from recession.

- If one also adopts the view that "combination inertia» (the man's job + the woman's job + local protectionism, etc.) is a structural change with greater influence than other demographic changes in favor of mobility ("the cohabitation transition»), the model will overestimate the future level of mobility in general.

The level of migration depends (see Holm and Öberg, 1984) to a large extent on factors unconnected with the labor market. On the other hand, labour market conditions may have more effect on migrations to and from individual areas. The geographical directions of net flows are probably influenced to a greater degree than is the level of migration by changes in regional labor demand.

Conceivable causes

\begin{tabular}{|l|}
\hline $\begin{array}{l}\text { Labor market: } \\
\text { extent } \\
\text { distribution } \\
\text { change }\end{array}$ \\
\hline $\begin{array}{l}\text { Changes in households: } \\
\text { co-migration } \\
\text { moving together } \\
\text { moving apart } \\
\text { moving away from home }\end{array}$ \\
\hline $\begin{array}{l}\text { Housing market: } \\
\text { extent } \\
\text { distribution } \\
\text { change }\end{array}$ \\
\hline
\end{tabular}

Causes according to the models

\begin{tabular}{|c|}
\hline $\begin{array}{c}\text { Labor market: } \\
\text { extent }\end{array}$ \\
\hline Co-migration \\
\hline $\begin{array}{c}\text { Exogenous supply, } \\
\text { unchanged out-migration, } \\
\text { commuting }\end{array}$ \\
\hline
\end{tabular}


In the table on p. 182 , we attempt to summarize some conceivable causes of migration, in addition to the causes incorporated in the CP and MS models.

The MS and HO models also contain a calculation of directed migrations between pairs of municipalities. The input data is a matrix which includes the historical (1981-82) migration between municipalities. Instead of constructing a model which derives the explanation for these flows externally, from a number of exogenous variables, the result - the observed migration pattern - is regarded as maximally "informative» in predicting the pattern of future migration. If the number of migrants between municipalities $\mathrm{A}$ and $\mathrm{B}$ diverges from the number to be expected if destination and origin were mutually independent, it is assumed that the factors underlying this divergence will retain the same force in the future.

The above assumption means that as much information as possible in the observed migration pattern must recur in the estimated future pattern. In the $M S$ model, the observed pattern is modified as little as possible, but in such a way that the summarized migration into and out of the municipality tallies with the new estimated values generated by the CP and MS calculations. It may be demonstrated that the "minimum information principle" corresponds to a reasonable statistical implication of the expression »as similar as possible». In other words, among all the possible alternative ways of classifying migrants, the one used is that which is least unlikely to result from a random disturbance. Moreover, it may be shown that the result is equivalent to that obtained by means of a simple iterative proportioning procedure.

The method thus generates a simple consistency calculation from the new estimated migration totals for each municipality, provided that no other conditions influencing the migration pattern's direction are changed. Ex post estimates for the period 1981-82 show that the largest error in the estimated number of migrants between two municipalities occurs in the migration totals calculated previously, i.e., the estimate CP and MS have in common.

The $\mathrm{HO}$ model is, in all essentials, based on the multiregional population projection model constructed by Rogers (1978) and presented at the 1982 Kungälv symposium (Holmberg, 1982). Briefly, the method may be characterized as a technique permitting the projection of a consistent migration pattern between pairs of municipalities. The population is divided into five age classes, but not by sex: this involves limitations in the fertility and mortality estimates, a flaw we may perhaps disregard in this context. Multiregional out-migration rates are calculated, using Markov technique.

$$
P(X)=\left[I+\frac{5}{2} M(X)\right]^{-1}\left[I-\frac{5}{2} M(X)\right] \quad X=\text { age }
$$

where $\mathrm{P}(\mathrm{X})$ and $\mathrm{M}(\mathrm{X})$ are $\mathrm{n} \times \mathrm{n}$ matrices in which

$$
M(x)=\left[\begin{array}{cccc}
{\left[M_{1 \hat{\delta}}(x)+\sum_{j \neq 1} M_{i j}(x)\right]} & -M_{21}(x) & \ldots & -M_{n 1}(x) \\
-M_{12}(x) & {\left[M_{2 \delta}(x)+\sum_{j \neq 2} M_{2 j}(x)\right]} & \ldots & -M_{n 2}(x) \\
\vdots & \vdots & & \\
\vdots & \vdots & & \vdots \\
\vdots & \vdots & & \\
-M_{1 n}(x) & -M_{2 n}(x) & \ldots & {\left[M_{n \hat{o}}(x)+\sum_{j \neq n} M_{n j}(x)\right]}
\end{array}\right]
$$

where $\mathrm{n}$ is the number of regions;

$\mathbf{M}_{\mathrm{i} \delta}(\mathrm{x})$ are age-specific mortality rates for region $\mathrm{i}$;

$\mathbf{M}_{i j}(x)$ are age-specific migration rates from region $i$ to region $j$; 
and $\mathrm{P}_{\mathrm{ij}}$, correspondingly, represents the probability of a person living in region $\mathrm{i}$ at period (1) surviving and living in region $\mathrm{j}$ five years later. The model thus employs transitions over five-year periods, which means that in its present state it can project forward only in time intervals of multiples of five years.

The method has certain shortcomings. It can, for example, be demonstrated that $\mathrm{P}_{\mathrm{ij}}$ may become negative in certain circumstances (when out-migration rates are high). If the model is used for large regions, as it is here, this hardly poses any problem. The weakness of the method is, rather, that it omits links between migration rates and other explanatory factors. The model postulates a constant multiregional migration pattern over a projection period of five, ten, etc., years, and calculates a pattern of directed migration on the basis of the population's initial age structure. As regards directed migration flows, the method thus has the same drawback as the MS-method. Unlike MS, it contains no rectrictions on total migration into a region. Both MS and CP calculate total out-migration by, in principle, the same method, namely trend projection. The difference is that in-migration and, thereby, net migration, are based on labor market trends in MS and $\mathrm{CP}$.

Compared with both the above methods, the $\mathrm{HO}$ model must be regarded as inferior for the purpose of explanation, in any case until a technique permitting labor market trends and migration rates to be linked can be devised. If the model is also to show the supply of labor, a sex distribution of the population is probably necessary. On the other hand, it might measure up to the other models when used for forecasting, at least in a short forecasting perspective.

\section{Comparison of results}

In the table below we shall outline the main results in terms of the population in 1990 and net migration during the period 1981-85.

\begin{tabular}{lccccc}
\hline Region & $\begin{array}{c}\text { Popu- } \\
\text { lation } \\
1980\end{array}$ & $\begin{array}{c}\text { Popu- } \\
\text { lation } \\
1990 \\
\text { CP }\end{array}$ & MS & HO & $\begin{array}{c}\text { Max. \% } \\
\text { diffc. } \\
\text { (1990) }\end{array}$ \\
\hline 1 Greater Stockholm & 1387 & 1437 & 1505 & 1452 & 4.9 \\
2 E. Central Sweden & 1030 & 1042 & 1028 & 1046 & 1.6 \\
3 S-E. Sweden & 774 & 767 & 754 & 764 & 1.7 \\
4 Greater Malmö & 419 & 423 & 426 & 415 & 2.6 \\
5 Rest of S. Sweden & 758 & 762 & 753 & 751 & 1.5 \\
6 Greater Gothenburg & 693 & 686 & 681 & 692 & 1.6 \\
7 Rest of W. Sweden & 944 & 933 & 938 & 949 & 1.7 \\
8 N-W. Central Sweden & 865 & 846 & 852 & 842 & 1.2 \\
9 Central Sweden & 534 & 518 & 497 & 522 & 4.7 \\
10 Central Norrland & 403 & 393 & 397 & 393 & 1.0 \\
11 Rest of Norrland & 511 & 495 & 502 & 505 & 2.0 \\
\hline Total & 8318 & 8303 & 8335 & 8331 & 0.4
\end{tabular}

For regions where the difference between the highest and lowest alternative amounts to nearly 5 percent, it is the MS which mentions a development which runs counter to previous trends. The »extreme values» of the MS reflect trend reversals in the demand for labor, constraints on unemployment rates and a high level of expected labor supply. On this point, the forecasts in CP tend to be trend projections, whereas the MS assessments are, rather, the results of assumptions 
of declining employment in the staple industries, and a high growth rate in, above all, the private white-collar sector, in the nation as a whole. Forecasts for Greater Malmö also display a relatively large deviation. The lower value in the HO alternative is a reflection of negative development during 1981-82, from which period the migration pattern data are taken.

In other respects the differences must be considered marginal. Since there are certain differences in assumptions about fertility and mortality, a comparison of net migration in 1981-85 may also be relevant.

\begin{tabular}{|c|c|c|c|c|}
\hline Region & $\mathrm{CP}$ & MS & $\mathrm{HO}$ & $\begin{array}{c}\text { Max. } \\
\text { diffe. }\end{array}$ \\
\hline 1 Greater Stockholm & 17,800 & 22,400 & 24,000 & 6,200 \\
\hline 2 E. Central Sweden & 3,100 & -300 & 2,800 & 3,400 \\
\hline 3 S-E. Sweden & 100 & -900 & $-2,100$ & 2,200 \\
\hline 4 Greater Malmö & 1,000 & 0 & $-2,500$ & 3,500 \\
\hline 5 Rest of S. Sweden & 5,800 & 8,400 & 2,200 & 6,200 \\
\hline 6 Greater Gothenburg & $-6,200$ & $-9,000$ & $-3,800$ & 5,200 \\
\hline 7 Rest of W. Sweden & 7,200 & 7,900 & 5,500 & 2,400 \\
\hline $8 \mathrm{~N}$-W. Central Sweden & 2,100 & 4,700 & - 900 & 5,600 \\
\hline 9 Central Sweden & $-6,400$ & $-14,000$ & $-4,800$ & 9,200 \\
\hline 10 Central Norrland & 300 & 2,400 & 0 & 2,400 \\
\hline 11 Rest of Norrland & $-9,300$ & $-5,700$ & $-6,000$ & 3,600 \\
\hline
\end{tabular}

The differences here are more striking: in four cases, the difference is greater than any forecasted absolute number, and the forecasts in these cases do not tally as to whether the figures are plus or minus. The large visible differences are, of course, primarily due to the low level of net migration as such. The difference between CP and MS is a result of divergent assumptions on labor demand and employment participation rates, whereas the $\mathrm{HO}$ alternative reflects actual developments in 1981-82.

\section{Conclusions}

Regional migration forecasts have up to now been prepared only in county planning, and such forecasts are still regarded as "official». Although in this paper we have dwelt somewhat on the defects of the different alternative models, we would claim that the presentation here of alternative assessments with at least partly divergent techniques is a strength. This applies primarily to the MS breakdown, which constitutes the first concerted attempt to co-ordinate a demographic, labor and migration forecast with a summarized one, using the same definitions and regional classification. The HO- model is perhaps somewhat outside the scope of such a comparison, in that it does not permit a discussion of the causes of forecast results which may be distinct. Indeed, this is the main reason for the efforts to co-ordinate $\mathrm{CP}$ and MS. The fact that the differences described above are nonetheless so small may be due partly to a failure to implement fully the regional labor estimates in the MS as had been planned.

Prior to future MS analyses, it is desirable to create a more direct link, within the framework of the model, between economic variables (prices, wages, etc.) and migration. This is, in fact, a prerequisite for an analysis of ways and means which would serve as a foundation for central policy decisions. 
We also consider it important for the CP- model to be developed further. Despite certain improvements, there is still no well thought-out causal connection to explain the gross migration level. Moreover, net changes in entire local labor markets are assumed to explain all net migration - the major gross changes in different labor sub-markets are not taken into account. However, a development of the model along these lines - with the retention of the detailed demographic classification - will probably mean that it assumes unmanageable proportions, even using modern computer technology. Switching to a microsimulation technique is a conceivable alternative, but it would require very extensive development work.

\section{References}

Holm, E. (1984). Åttiotalets flyttningar. ERU.

Holm, E. and Öberg, S. (1984). Hushållsförändringar som förklaring till flyttingar. SOU 1984: 1, Stockholm.

Holmberg, I. (1984). Model migration schedules - the case of Sweden. In: The Sixth Scandinavian Demographic Symposium 16-19 June 1982 in Kungälv, Sweden. Studies in Migration. Scandinavian Population Studies 6: 1. The Scandinavian Demographic Society, Stockholm.

Hårsman, B. and Schéele, S. (1984). Household projections with a reconciliation of demographic and economic factors. In: Scandinavian Population Studies 6: 1. The Scandinavian Demographic Society, Stockholm.

- Holmberg, I. and Schéele, S. (1984). Bostadsbyggandet till år 2000. Bostadsdepartementet, Ds Bo 1984: 6, Stockholm.

Ledent, J. (1981). Statistical analysis of regional growth. IIASA WP-81-128.

Lindström, H. and Strandberg, M. (1983). Det regionala arbetskraftsutbudet 1990, teknisk rapport, SCB 1983, Stockholm.

Rogers, A. and Willekens, F. (1978). Spatial population analysis: methods and computer programs, IISA RR-78-18.

Statens offentliga utredningar. (1984). Långtidsutredningen 1984: Regional analys. SOU 1984: 6, Stockholm.

Strömqvist, U and Karlqvist, A. (1982). Var kommer krisen? SIND 1982: 3.

Snickars, F. (1981). Regional analys i LU-80. Ekonomidepartementet. Stockholm.

Stålberg, L. and Hedlund, A. (1984). Various forecasting methods and their uses. In: Scandinavian Population Studies 6: 1. The Scandinavian demographic Society, Stockholm.

Öberg, S. (1984). Interregional migration. In: Scandinavian Population Studies 6: 1. The Scandinavian Demographic Society, Stockholm. 